\title{
Urine testing for HPV: rationale for using first void
}

\author{
Alex Vorsters researcher ${ }^{1}$, Pierre Van Damme professor ${ }^{1}$, Gary Clifford cancer epidemiologist ${ }^{2}$ \\ ${ }^{1}$ Faculty of Medicine and Health Sciences, Centre for the Evaluation of Vaccination, Vaccine and Infectious Disease Institute, University of Antwerp, \\ 2610 Antwerpen (Wilrijk), Belgium; ${ }^{2}$ Infections and Cancer Epidemiology Group, International Agency for Research on Cancer, Lyon, Cedex 08, \\ France
}

We agree that urine testing for human papillomavirus (HPV) in cervical cancer screening needs further evaluation, and that the heterogeneity of urine sampling and testing protocols should be resolved. ${ }^{12}$ In particular, attention should be given to the rationale and evidence for using first void urine for HPV testing. During urination, urine is contaminated by impurities, including mucous and the debris of exfoliated cells from the vagina, cervix, and uterus. The initial flow of urine collects most of this debris, which is why in women with cervical HPV infection the first collected part of a urine void contains more HPV DNA than subsequent parts, as concluded by Pathak and colleagues ${ }^{1}$ and confirmed by our recent research. ${ }^{3}$ Furthermore, our unpublished findings suggest that the volume of collected first void urine and time of collection (first urination of the day $v$ subsequent urinations) are additional sources of variation. Because of the importance of the first void urine for HPV detection, a collection device has been developed that ensures immediate mixing of the first void urine with a preservation medium, while allowing the rest of the urine to exit the device unhindered (avoiding the need to interrupt the flow). This device was shown to have excellent acceptability in 1000 young women in Bhutan and Rwanda (Gary Clifford, unpublished data).

In summary, standard criteria for volume, collection, storage, extraction, and HPV testing are essential to maximise HPV detection from urine, and the collection of the first void is a key step.

Competing interests: AV and PVD are cofounders of Novosanis, a spin-off company of the University of Antwerp, responsible for valorisation of the urine collection device. GC declares no conflicts of interest.

Full response at: www.bmj.com/content/349/bmj.g5264/rr/769123.

1 Pathak N, Dodds J, Zamora J, Khan K. Accuracy of urinary human papillomavirus testing for presence of cervical HPV: systematic review and meta-analysis. BMJ 2014;349:g5264. (16 September.)

2 Kitchener HC, Owens GL. Urine testing for HPV. BMJ 2014;349:g5542. (16 September.)

3 Vorsters A, Van den Bergh J, Micalessi I, Biesmans S, Bogers J, Hens A, et al. Optimization of HPV DNA detection in urine by improving collection, storage, and extraction. Eur J Clin Microbiol Infect Dis 2014; published online 12 Jun. 